\title{
The Emergence of New Trophic Levels in Eco-evolutionary Models with Naturally-bounded Traits
}

\author{
Roger Cropp \\ School of Environment and Science, Griffith University, Gold Coast, Qld, 4215, Australia \\ Centre for Applications in Natural Resource Mathematics, School of Mathematics and Physics, The University of \\ Queensland, St Lucia, Qld 4072 \\ John Norbury \\ Mathematical Institute, University of Oxford, Andrew Wiles Building, ROQ, Woodstock Road, Oxford OX2 6GG, UK
}

\begin{abstract}
Ecosystems and food webs are structured into trophic levels of who eats whom. Species that occupy higher trophic levels have less available energy and higher energetic costs than species at lower trophic levels. So why do higher trophic levels exist? What processes generate new trophic levels? We consider a heuristic eco-evolutionary model based on simple Lotka-Volterra equations, where the evolution of traits is described by a generalisation of Lande's equation. The 15 transition from competition to predation in this simplest of models is a successful, safe strategy for a population, and suggests a propensity to develop new trophic levels may be an inherent property of ecosystems.

Numerical simulations with a more complex eco-evolutionary model of interacting plant and herbivore populations display the emergence of a new trophic level as an alternative to continued 20 competition. These simulations reveal that new trophic levels may arise naturally from ecosystems because a robust strategy for a population in the presence of a strong competitor that could dominate or potentially extinguish them, is to predate upon the competitor. The same properties that make the competitor strong make it an ideal prey, suggesting the rubric that it is better to eat a strong competitor than to continue competing.
\end{abstract}

25 Keywords: eco-evolutionary model, emergence of new trophic levels, $\gamma$-distributed traits, natural trait bounds, emergence of carnivory

\section{Introduction}

The creation of new trophic levels in ecosystems is a fundamental component of the development of the complex food webs that are evident in many ecosystems today (Landi et al.

30 2018). The complexity-stability debate, essentially whether complexity begets stability or vice versa (Odum, 1953; May, 1972), has yet to be unequivocally resolved, but the fact that ecosystems develop complexity is apparent in many environments. The geological record suggests that trophic levels that are destroyed in mass extinctions eventually become re-established, albeit slowly (Sahaney and Benton, 2008). The consensus is that ecosystems only have a few trophic 
${ }_{35}$ levels because the energy available to the occupants of a trophic level decreases, and the respiration costs of existing at a trophic level increase, as the trophic level increases (Lindeman 1942, Bonhommeau et al., 2013). This begs the question of why new trophic levels evolve what benefit is there for a population to stop competing with other populations within a trophic level and to start eating its competitors, if there are fundamental energetic constraints associated 40 with moving to a higher trophic level (Hastings and Conrad, 1979)?

This work considers the development of new trophic levels that result when a population ceases competing with, and begins predating on, its erstwhile competitor. This process is thought to be a key contributor to the development of biodiversity as the role of predation is increasingly recognised for its effect in shaping ecosystems (Terborgh, 2015). We examine this from two per-

45 spectives: generic population interaction considerations based on dynamical system properties of competition and predator-prey interactions; and a specific computed example of the mechanics of how a competitor adapts to become a predator. The first perspective provides a rationale for why the process examined in the second perspective exists. First, however, we provide an overview of the processes involved in dynamical eco-evolutionary modelling.

The development of new trophic levels is related to food-chain length, with ecosystem size often considered a more important determinant of food-chain length than resource availability or disturbance (Post, 2002). Although models exist that can describe food-web structure quite accurately, there remains little understanding of how these structures develop (Loeuille and Loreau 2005) or of how organism-level evolution relates to community structure (Romanuk et al. 2019). Relating the traits of individual organisms to ecosystem processes remains a challenge (Hawlena and Schmitz, 2010), although it is recognised that adaptive behaviour can be an important structural determinant of food webs (Kondoh, 2003, Loeuille and Loreau, 2005 Thompson et al. 2012). Recent work has, however, shown that ecosystem-level properties may constrain organism-level evolution (Cropp and Norbury, 2019a).

${ }_{60}$ The variance of traits in a population may play a significant role in the adaptive behaviour of populations (Lande, 1979) and the subsequent development of ecosystem structure. Trait variance may also be affected by mutations in the genome, and although the role of mutation bias in adaptive evolution remains unclear, it seems that the impression that directed mutations can drive adaptive evolution is misleading (Svensson and Berger. 2019).

65 New trophic levels may be created in two ways: an autotroph may become a heterotroph (for example, a planktonic algae ceases to consume inorganic nutrient and commences feeding on organic matter (Bell, 2012), passing through a stage called mixotrophy (Edwards, 2019) or a heterotroph changes the trophic level that it feeds on (for example, a herbivore becomes a carnivore). The abandonment of autotrophy in favour of heterotrophy, when the environment has 70 changed to favour the latter, has been demonstrated in laboratory experiments in mixotrophic algae that repurpose cellular machinery developed for autotrophy to heterotrophy (Bell, 2012). The efficacy of eco-evolutionary processes to simulate how populations may transition from autotrophy to heterotrophy was recently demonstrated using an eco-evolutionary model (Cropp and Norbury, 2019b). We focus on the second case here, and consider how a herbivore becomes 75 a carnivore.

Eco-evolutionary modelling attempts to determine how the mean values of a population's traits change in response to the effects of other populations and the environment upon the population's fitness (Nuismer 2017), and has been applied to many ecological systems (Abrams 1999, Abrams and Matsuda, 2004; Chesson, 2000; Cortez and Ellner, 2010; Cortez and Patel,

so 2017). Recent work has shown that eco-evolutionary processes can have profound effects on the structure, complexity and dynamical properties of food webs (Merico et al., 2014, Gibert and 
Yeakel. 2019).

The equation derived by Lande (1976) lies at the heart of eco-evolutionary modelling:

$$
\frac{d \bar{z}}{d t}=h^{2} \sigma^{2} \frac{\partial \ln \bar{W}}{\partial \bar{z}} .
$$

Lande's equation relates the rate of change in the average value $\bar{z}$ of a population trait $z$ (for

85 example, body size or food assimilation efficiency) to the additive heritability of the trait $h^{2}$, the variance of the trait value distribution $\sigma^{2}$, and the sensitivity of the average population fitness $\bar{W}$ to the average trait mean value. Lande's equation may be coupled to an ecological model to construct the eco-evolutionary model. The ecological models are usually simple, heuristic models of population interactions, often Lotka-Volterra models that use carrying capacity to

90 represent the constraints of the environment on the populations and to ensure sensible solutions (Abrams, 2000, Birand and Barany, 2014).

In deriving his equation, Lande assumed that trait values $z$ were normally distributed in the population, and that consequently the trait variance $\sigma^{2}$ was independent of the trait mean $\bar{z}$. However, recent work has suggested that many traits have non-normal distributions (Gaedke and

95 Klauschies, 2017). This suggests that trait distributions may be responding to constraints upon the values they may take, and that a bounded distribution such as a gamma-distribution may be a more appropriate assumption. In fact, bounded trait distributions have been used in several eco-evolutionary models (Abrams, 1999; Abrams and Matsuda, 2004; Cortez and Ellner, 2010 Cortez and Patel. 2017). Others have considered bounded tradeoffs in fluctuating environments Kremer and Klausmeier (2013), or have evolved the moments of the distributions separately (Merico et al. 2009).

While it is recognised that trait bounds prevent runaway evolution in systems with disruptive selection (Cortez and Patel. 2017), it is not clear which traits should have bounds, and what the values of the trait bounds should be. A resource-based approach to constructing ecological models (Cropp and Norbury, 2015, 2019c) has, among others, the advantage that the ecosystem equations naturally define bounds upon many trait values, particularly those that relate to population interactions (Cropp and Norbury, 2019a b). These naturally occurring trait bounds, which arise even in simple Lotka-Volterra models, suggest that traits are better represented by (half- or fully-) bounded rather than normal distributions. The resource-based approach to constructing ecological models requires that every population in the model has its limiting resource(s) explicitly represented and dynamically tracked, and that limiting resources, and consequently populations, are finite. It may prevent runaway evolution by placing upper, lower, or both bounds on trait means, but may also identify trait means that are effectively unbounded by population dynamics. Such bounds would in practice be constrained by physiological constraints, but these constraints do not necessarily constrain the eco-evolutionary dynamics.

The final assumption required for eco-evolutionary modelling is the measure $\bar{W}$ the average fitness of individuals in the population. The choices for $\bar{W}$ are many and varied. Eco-evolutionary models that simulate very specific scenarios, such as the coevolution of the average rostrum length of a weevil population and the average pericarp thickness of a plant population (Nuismer 2017), require very specific definitions of fitness. Other models consider more general, or abstract aspects (Cortez and Patel, 2017) of eco-evolutionary interactions with appropriately more general definitions of fitness.

A common assumption when considering heuristic models of eco-evolution, where much detail is abstracted away, is to use a simple generic description of the average fitness of the individuals in a population based on the population net per capita growth rate (Abrams et al. 
1993, Fussmann et al. 2003, Cortez, 2016, Pastor, 2017). This is represented by the function $f_{i}$ in the canonical-form ecosystem model (Kolmogorov, 1936):

$$
\frac{d x_{i}}{d t}=x_{i} f_{i}\left(x_{1}, x_{2}, \ldots, x_{n} ; z_{1}, z_{2}, \ldots, z_{m} ; R_{i}\right),
$$

that describes how the population $x_{i}$ changes within an ecosystem of $n$ populations $x_{1}, x_{2}, \ldots, x_{n}$ that collectively have $m$ traits $z_{1}, z_{2}, \ldots, z_{m}$ and survive on resource(s) $R_{i}$. The resource(s) $R_{i}$ may be a pool of inorganic nutrient in the case of autotrophs, or other populations in the case of heterotrophs (see $\$ 2$ for details).

One approach is to define the average fitness of a population $x_{i}$ as the exponential of its net growth function $f_{i}$, that is, the realised response of the population to changes in the populations it interacts with, their traits, and the environment (Vasseur et al. 2011; Osmond et al., 2017, Cropp and Norbury 2019a b ). Defining $\bar{W}_{i}=e^{f_{i}}$ leads to the sensitivity of the average population fitness term:

$$
\frac{\partial \ln \bar{W}_{i}}{\partial \bar{z}_{j}}=\frac{\partial f_{i}}{\partial \bar{z}_{j}} .
$$

The derivation of Lande's equation assuming $\gamma$-distributed traits (which are bounded on one side), where the variances of the distributions are functions of the means and are defined in terms of the bounds, is provided in Cropp and Norbury (2019a). Lande's equation (1) becomes, for bounded traits $z_{j}$ of a population $x_{i}$ :

$$
\begin{aligned}
& \frac{d \bar{z}_{j}}{d t}=h_{j}^{2}\left(\frac{z_{j}^{U}-\bar{z}_{j}}{\beta_{j}}\right) \frac{\partial f_{i}}{\partial \bar{z}_{j}}, \text { for an upper-bounded trait }\left(\bar{z}_{j}<z_{j}^{U}\right), \\
& \frac{d \bar{z}_{j}}{d t}=h_{j}^{2}\left(\frac{\bar{z}_{j}-z_{j}^{L}}{\beta_{j}}\right) \frac{\partial f_{i}}{\partial \bar{z}_{j}}, \text { for a lower-bounded trait }\left(\bar{z}_{j}>z_{j}^{L}\right) ;
\end{aligned}
$$

we also use

$$
\frac{d \bar{z}_{j}}{d t}=h_{j}^{2}\left(\frac{\left(z_{J}^{U}-\bar{z}_{j}\right)\left(\bar{z}_{j}-z_{j}^{L}\right)}{\beta_{j}\left(z_{j}^{U}-z_{j}^{L}\right)}\right) \frac{\partial f_{i}}{\partial \bar{z}_{j}}, \quad \begin{gathered}
\text { for a trait bounded both above } \\
\text { and below }\left(z_{j}^{L}<\bar{z}_{j}<z_{j}^{U}\right)
\end{gathered},
$$

where $\beta_{j}$ is a parameter that describes the shape of the $\gamma$-distribution of $z_{j}$. A key feature of this approach is that the evolutionary bounds $z_{j}^{L}$ and $z_{j}^{U}$ are defined by the ecological condition for sensible populations, $\left.f_{i}\right|_{\max \left(R_{i}\right)}>0>\left.f_{i}\right|_{\min \left(R_{i}\right)}$, that ensures populations are viable but not immortal (see \$2 . Note this is similar to Abram et al.'s approximation (Abrams et al., 1993).

The assumption that some traits are $\gamma$-distributed means that the mean trait value and the trait variance evolve simultaneously, and is consistent with Fisher's view (Fisher, 1930; Price 1972) that any moment of trait distributions may evolve (Walsh and Lynch 2018 p 147), and also Price's theorem (Price, 1970). In the course of a population's evolution, trait means may reside close to their boundaries as the population maximises its fitness, so that trait distributions are not normal and are appropriately described by $\gamma$-distributions.

Bounded distributions have previously been used in eco-evolutionary studies (for example (Kremer and Klausmeier, 2013, Klauschies et al., 2018)), as have bounded versions of Lande's equation (for example (Abrams, 1999, Abrams and Matsuda, 2004, Chesson, 2000, Cortez and Ellner, 2010, Cortez and Patel, 2017)). However, a key difference between our approach and that 
155 of previous studies is that the bounds for the evolutionary equations specified by the assumption of $\gamma$-distributed traits are defined explicitly by the general principles (see $\$ 2$ below) that apply to the ecological equations.

Note that Lande (1976) used the notation for the narrow sense heritability (Fisher, 1930; Kempthorne, 1957) in his equation (1) but defined the heritability of a trait more generally as " $h^{2}$ is the realized heritability of the character, which is determined by the genetic system, the breeding structure of the population and the environment, and may change during the course of evolution." This is more in line with the historical use of heritability in the context of the selection response (Nicholas, 1987). The bounded versions (4) interpreted in the narrow sense suggest that heritability will go to zero if the variance of the trait in the population goes to zero,

165 stopping evolution. While the bounds are theoretical limits that it is unlikely a population would reach in practice, so that the trait variance and hence heritability would become small but never reach zero, we do not labour this point and allow the exponential solutions to the ode system to represent this property.

In this paper we take an eco-evolutionary approach to the question of why and how new 170 trophic levels become established in ecosystems. We first consider the generic properties of these models that result from the assumptions above, and consider what aspects of these properties provide sufficient advantages to populations for them to abandon competition and start eating their competitors. We describe in $\$ 2$ underlying ideas via a simple example - this may be skipped if the reader is solely interested in simulation outcomes. The purpose of $\$ 2$ is to use a heuristic

175 food-web model to explain why there is a basic tendency for evolution to drive competitors to generic strategies that create new trophic levels.

We then develop a more complex (but still heuristic) eco-evolutionary model in $\S 3$ and consider several scenarios of eco-evolution. The eco-evolutionary simulation model is stated in $\$ 3$ and the results of the simulations are provided in $\$ 5$

\section{General Principles}

A simple model built upon the generic assumptions outlined above provides some insights into the general properties of these eco-evolutionary models. Consider a simple, generic model of a population $x$ that consumes a resource $R$, that may be an inorganic nutrient or another population (plant or animal), where $x$ is preyed upon by another population $y$, and experiences natural mortality. We describe the dynamics of the population $x$ with an ordinary differential equation:

$$
\frac{d x}{d t}=x f_{x}(x, y, r)=x(g r-p y-m)
$$

where $g$ is the maximum per capita growth rate of $x, p$ is the rate at which $y$ predates upon $x$, and $m$ is the natural, density-independent mortality rate of $x$. We have written the equation (6) using the usual convention that the growth, grazing and mortality parameters that describe the mean values of the $x$ population's traits $(g, p$, and $m$ ) are positive, and their effect on the growth of $x$ is evidenced by the sign of each term.

We measure both populations and the resource in the same currency, usually the fraction of the limiting resource that they contain (i.e. the mass of limiting nutrient in populations $X$ and $Y$, and the resource pool $R$, are scaled by the total mass $X+Y+R$ of limiting nutrient in the 195 ecosystem, so that $x=X /(X+Y+R), y=Y /(X+Y+R)$, and $r=R /(X+Y+R))$. This assumes that the limiting resource is completely recycled within the system, a common property of many 
ecosystems, that typically recycle over $90 \%$ of their limiting nutrient (Vitousek and Matson 2012). Complete recycling provides the relation $x+y+r=1$, which defines the maximum and minimum values that the populations and the resource fractions may take: $0 \leq x, y, r \leq 1$ with $0 \leq x+y \leq 1$.

We impose ecological axioms on the model to ensure that the population $x$ is sensible (Cropp and Norbury, 2015) and consistent with the properties seen in real ecosystems. First we ensure that the population is viable, that is, the population can grow when its resources are the maximum possible, in this case $r=1$. This requires that $f_{x}$, the net per capita growth rate of $x$, is positive when $r=1$. We write this in symbols for equation (6) as:

$$
\left.f_{x}\right|_{\{r=1, x=0=y\}}>0 \Rightarrow g-m>0 \Rightarrow g>m .
$$

We also ensure that $x$ is not immortal, by requiring that it cannot grow (i.e. $f_{x}<0$ ) when it has no resources $(r=0)$. When $r=0$ we consider the options $x=1, y=0$ and $x=0, y=1$, as each provides a different constraint:

$$
\begin{aligned}
& \left.f_{x}\right|_{\{r=0, x=1, y=0\}}<0 \Rightarrow-m<0 \Rightarrow m>0, \\
& \left.f_{x}\right|_{\{r=0, x=0, y=1\}}<0 \Rightarrow-p-m<0 \Rightarrow p+m>0 .
\end{aligned}
$$

Together, (7) and (8) provide the trait value constraints:

$$
0<m<g \text {, and }-m<p .
$$

Note that $0<g, p, m$ by the convention used to write equation (6), so the constraint $-m<p$ is redundant, but this is not always the case. These constraints on the values that the trait means may take require that we write the equations that describe how the trait means evolve assuming that the trait values have bounded distributions.

\subsection{An eco-evolutionary rubric}

We derive the equation for the trait mean $\bar{z}$ for the cases $p$ and $m$ for the ecological model (6) for each growth rate $g>0$ that may be chosen, using the fitness sensitivity definition of (3) and the bounded forms of (4), and couple them to the ecological model, to provide the heuristic eco-evolutionary system:

$$
\begin{aligned}
& \frac{d x}{d t}=x f_{x}(x, y, r)=x(g r-p y-m), \\
& \frac{d p}{d t}=-h_{p}^{2}\left(\frac{p}{\beta_{p}}\right) y, \\
& \frac{d m}{d t}=-h_{m}^{2}\left(\frac{(g-m) m}{\beta_{m} g}\right),
\end{aligned}
$$

where $h_{i}^{2}, i=p, m$ are the additive heritabilities of the traits, and the $\beta_{i}, i=p, m$ are parameters that define the shape of the respective $\gamma$-distributions. Here, the variances of the $\gamma$-distributions are given by the expressions within the large brackets in the equations for $\frac{d p}{d t}$, and $\frac{d m}{d t}$. Note that the parameters $p, m$ are the means of the distributions of the traits. 
The simple model (10) provides insight into the outcomes we might expect from more sophisticated eco-evolutionary models. As the heritabilities and the variances are always positive, the direction of evolution of each trait mean is determined by the sensitivity of the population fitness to the average trait value; in simple models this often has a fixed sign, but may vary in more complex models. Here, the maximum per capita growth rate $g$ can be chosen at any level, while the predation rate $p$ and the mortality rate $m$ always decrease: $p$ and $m$, are bounded by zero, which controls the values they can ultimately take. It appears natural that a population should evolve, for each (arbitrarily large) growth potential, to minimise its mortality and vulnerability to predation. This raises the question, is $g$ very large an ecologically realistic property, given that the fraction of the resource that the population can accumulate is bounded, with $0 \leq x \leq 1$ ?

Somewhat surprisingly, a theoretically unbounded maximum per capita growth rate $g$ is a legitimate property for a population in an ecosystem model (although in the real world physiological constraints, which are not included in this model, would place bounds on how fast a population could grow). This becomes evident when examining the location of the $f_{x}=0$ isocline of the population, which separates the region of the ecospace in which the population can grow from the region in which it can't. The $x$ axis intercept of the isocline is $x=(g-m) / g$, which tends to 1 as $m \rightarrow 0$ irrespective of the value of $g$. Similarly, the $y$ intercept of the isocline, $y=(g-m) /(g+p)$, also tends to 1 as $m$ and $p$ tend to zero, irrespective of the value of $g$, and can never exceed 1 (Figure 1 (a)).
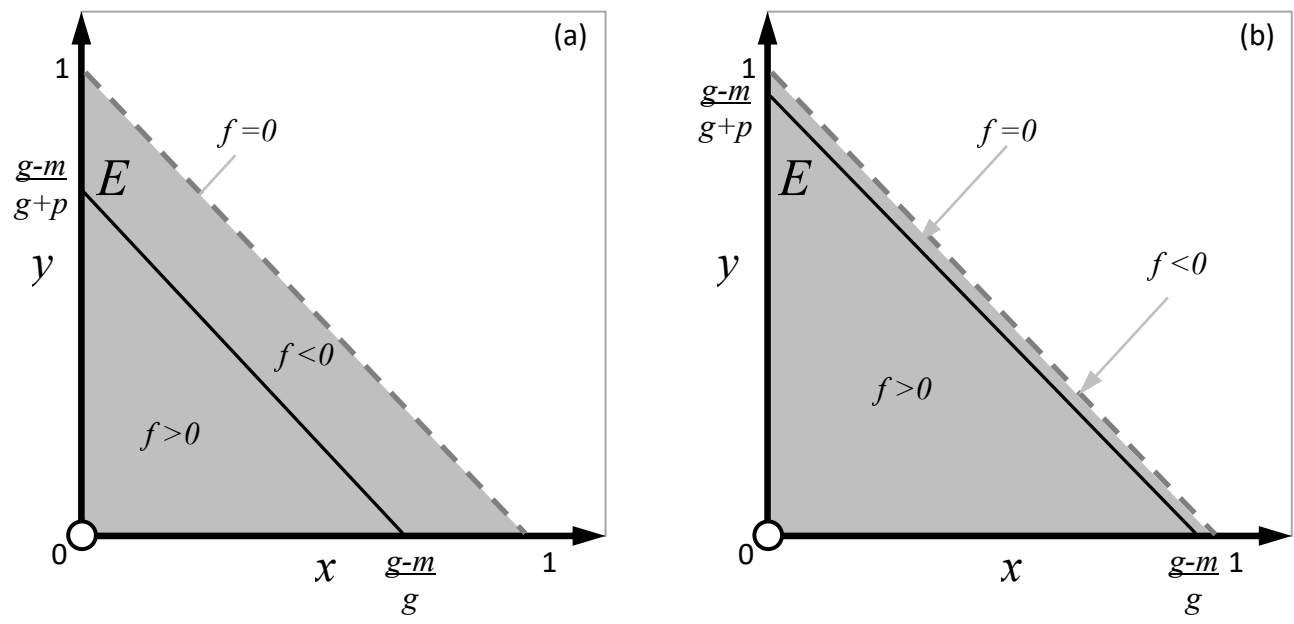

Figure 1: Diagrams of general attributes of eco-evolutionary models typified by (6) and (10). All ecologically valid solutions exist in the space $E \equiv\{0 \leq x \leq 1,0 \leq y \leq 1,0 \leq x+y \leq 1\}$ which is shown as the grey shaded area. The ecologically valid solutions are bounded by $x=0$ (the $y$ axis), $y=0$ (the $x$ axis), and $x+y=1$ (the dashed line). The white dots indicate unstable equilibrium points and the black dots indicate stable equilibrium points. (a) The $f=0$ isocline of a population divides the ecospace into a region where the population can grow $(f>0)$ and a region where it can't $(f<0)$. (b) The effect of a population increasing its fitness is to maximise the region of the ecospace in which it can grow. It does this by moving its $f=0$ isocline as far from its point(s) of maximum resource as possible. See Cropp and Norbury (2019a) for details.

The outcome of evolution in the simple model $[10$ can be summarised neatly by the rubric 
that evolution works to move the zero isocline of the population as far from its maximum resource as it is possible to go in the ecospace (Figure 1 (b)). This means that the population maximises the region of the ecospace in which it can grow, simultaneously minimising the region in which it can't. This outcome is consistent with Tilman's $R^{*}$ theory (Tilman, 1977, 1981), which predicts that the winner of competition between populations that compete for the same resource will be the population that leaves the least resource for its competitor when it is at its equilibrium.

\subsection{Implications of the rubric}

If we extend the above argument to two generic populations $x$ and $y$ that compete for the same resource, then these populations will evolve to have zero isoclines that are as near to the zero resource state as physiology allows, and are nearly parallel (Figure 2(a)). This is a parlous state for both competitors, as small variations in their trait values can led to dramatically better or worse outcomes, as the location of the coexistence equilibrium point (the black dot) moves substantially in response to small changes in the locations/angles of the isoclines. A population may be sent to extinction by relatively minor changes in a competitor's properties, as described by $R^{*}$ theory (Tilman, 1981 ).

A safe trophic strategy for a population in competition with another population for a resource, in the absence of alternative resources, that minimises adverse impacts on its equilibrium share of resource due to adaptations by its competitor, is to eat its competitor. In Figure 2 population $x$ 's change from competitor to predator is represented by rotating the $f_{x}=0$ isocline clockwise. The change from competition with, to consumption of, another population is a robust one, as a stable equilibrium point that advantages the consuming population is maintained throughout the transition. As the emerging predator's (in this case $x$ 's) isocline rotates ( $f_{x}$ in Figure $2(\mathrm{~b})$ ), its equilibrium population size becomes less sensitive to changes in its competitor $(y)$, which is constantly co-evolving to allow it to compete more successfully.

Once the competitor $x$ has become a predator, all the attributes of its now prey $y$, that previously made it a formidable competitor, now make it an ideal prey. This is a key property for the new predator, which after giving up autotrophy, cannot survive in the absence of its prey. The

270 predator now has the potential to, as our rubric suggests, adapt to this new trophic interaction by maximising its region of positive growth in the ecospace. It does this by sliding its $f_{x}=0$ isocline as far from its maximum resource $y=1$ as possible. This produces the outcome shown in Figure 2(b), which allows the new predator to maximise its own population. This transition from competitor to predator produces a fundamental change in the structure of the food web.

This simple result suggests a profound hypothesis - that the basic properties of ecosystems in which populations adapt to other populations (i.e. most ecosystems) primes them to increase the number of trophic levels, that is, there is a natural tendency of food webs to accumulate increasing numbers of trophic levels. This theoretical result is obviously tempered by the physiological constraints of the populations, their environments, and the greater energy costs of carnivory, but provides a useful lens through which to view how ecosystems change. Cropp and Norbury (2019b) recently demonstrated the emergence of heterotrophy in a simple eco-evolutionary simulation model of plankton; we now consider the emergence of carnivory in a slightly more complex eco-evolutionary simulation model.

\section{The Eco-evolution Model}

We consider a simple, heuristic eco-evolution model based upon the classic Lotka-Volterra competition model, written in the Conservative Normal framework (Cropp and Norbury, 2015. 

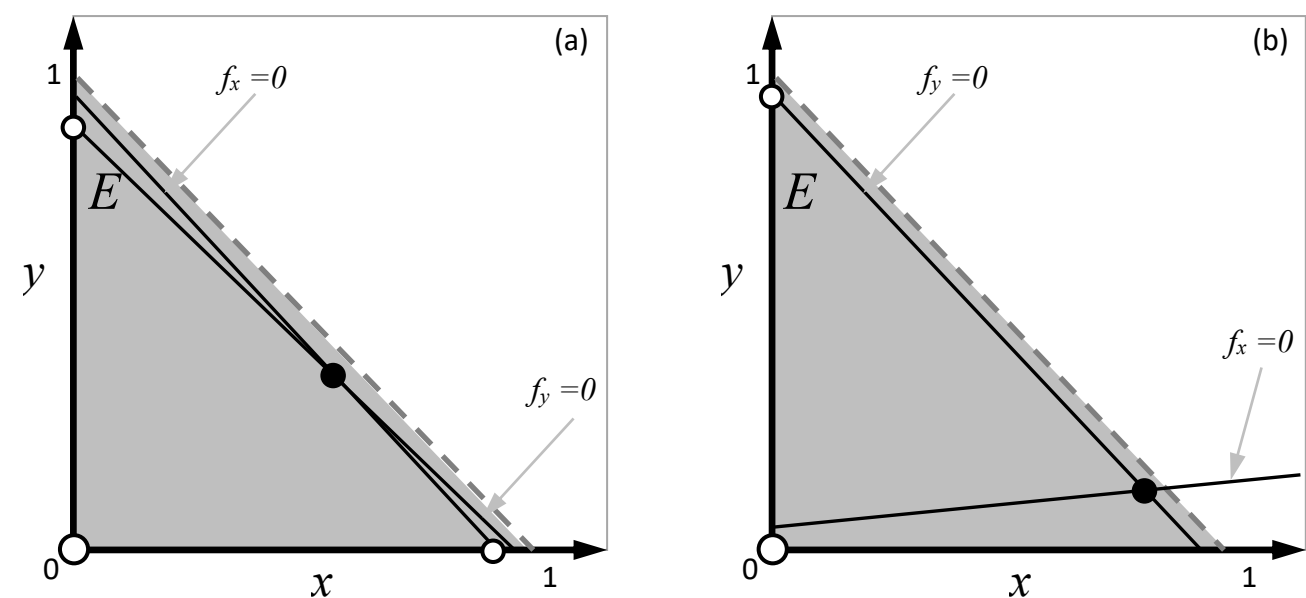

Figure 2: Two populations $x$ and $y$ that compete for the same resource $r$ will evolve to have near-parallel zero isoclines near their zero resource boundary, in this case $x+y=1 \Rightarrow r=0$, as shown in (a). The eco-evolutionary outcome after $x$ switches resource from competing with $y$ for $\rho$ to consuming $y$ instead of $r$ is shown in (b). The change of resource is achieved by rotating the $f_{x}=0$ isocline clockwise, after which resource use is optimised by sliding the $f_{x}=0$ isocline down the $y$ axis. Details as for Figure 1 The location of the stable coexistence point favours $x$, and is now less sensitive to variations in the slope of the $f_{y}=0$ isocline.

2019c), coupled to Lande's equations that assume $\gamma$-distributed traits. Lotka-Volterra models are sometimes criticised for their lack of much connection to biological reality, however, these models are linear approximations to all models May (1973), and what they lack in realism they more than make up for in heuristic value, as many nonlinear ecosystem models are functioning in their linear part much of the time. As the objective of this work is to explore generic properties of predator-prey interactions, the heuristic benefits of the Lotka-Volterra model outweigh the limitations of their inherent abstractions.

Three interacting populations are modelled: an autotroph (for example, a plant) $x_{1}$ that consumes an inorganic nutrient $N$, and is grazed upon by two competing heterotrophs (for example, herbivores) $x_{2}$ and $x_{3}$. Each population and the nutrient pool are measured in terms of their fractions of the limiting nutrient cycling in the food web (as described in $\$ 2$ : 


$$
\begin{aligned}
\frac{d x_{1}}{d t} & =x_{1} f_{x_{1}}\left(x_{1}, x_{2}, x_{3}, N\right)=x_{1}\left(r_{1} N-\hat{a}_{11} x_{1}-\hat{a}_{12} x_{2}-\hat{a}_{13} x_{3}\right), \\
& =x_{1}\left(r_{1}-\left(r_{1}+\hat{a}_{11}\right) x_{1}-\left(r_{1}+\hat{a}_{12}\right) x_{2}-\left(r_{1}+\hat{a}_{13}\right) x_{3}\right), \\
& =x_{1}\left(r_{1}-a_{11} x_{1}-a_{12} x_{2}-a_{13} x_{3}\right), \\
\frac{d x_{2}}{d t} & =x_{2} f_{x_{2}}\left(x_{1}, x_{2}, x_{3}\right)=x_{2}\left(r_{2}-a_{21} x_{1}-a_{22} x_{2}-a_{23} x_{3}\right), \\
\frac{d x_{3}}{d t} & =x_{3} f_{x_{3}}\left(x_{1}, x_{2}, x_{3}, a_{32}, \rho\right)=x_{3}\left(r_{3}-(1-\rho) a_{31} x_{1}-\rho a_{32} x_{2}-a_{33} x_{3}\right), \\
\frac{d a_{32}}{d t} & =-h_{a_{32}}^{2}\left(\frac{a_{32}+a_{23}}{\beta_{a_{32}}}\right) \rho x_{2}, \\
\frac{d \rho}{d t} & =h_{\rho}^{2}\left(\frac{(1-\rho) \rho}{\beta_{\rho}}\right)\left(a_{31} x_{1}-a_{32} x_{2}\right) .
\end{aligned}
$$

Note that we have substituted the conservation of mass condition $N=1-x_{1}-x_{2}-x_{3}$ in the $d x_{1} / d t$ equation to eliminate $N$ and defined the new parameters $a_{11}=r_{1}+\hat{a}_{11}, a_{12}=r_{1}+\hat{a}_{12}$,

\subsection{Equilibrium Points}

The ecological sub-model has eight potential equilibrium points $C_{0}-C_{7}$, the locations of which are described in detail in Appendix $\mathrm{A}$. Two of these equilibrium points, $C_{2}$ and $C_{3}$ are al-

All the terms in the population interaction matrix $A=a_{i j}$ for $i, j=1,2, \ldots, n$ of a LotkaVolterra competition model are by convention positive. As all competing populations have a detrimental effect on the other populations in the model, either directly or indirectly, competition is represented by negative signs in front of each $a_{i j}$. Non-competitive interactions in these models are then represented by negative terms in the population interaction matrix $A$.

The natural mortalities of $x_{1}, x_{2}$, and $x_{3}$ are captured by $a_{11}, a_{22}$, and $a_{33}$ respectively, while $a_{21}$, and $a_{31}$ are grazing parameters of $x_{2}$ and $x_{3}$ respectively upon $x_{1}$. These parameters have negative signs as shown in Table 1 so they function as benefits to $x_{2}$ and $x_{3}$. Detailed descriptions of the role of each of the model parameters, and the values used to draw the figures, are provided in Appendix D.

While all the other parameters are constants, $a_{32}$ and $\rho$ are the mean values of feeding traits of the individuals that make up the $x_{3}$ population. Consistent with derivation of Lande (1976) and following the approach of Cortez and Patel (2017), the frequency dependence of the population's fitness to the population's traits is represented by the sensitivity of the population mean fitness $\bar{W}$ to the mean trait value $\bar{z}$ (equations (1) and (3)).

A change in trophic strategy in the context of this model must be achieved through the modification of an existing attribute. The transition from herbivory to carnivory must then utilise the same metabolic pathways, a potential common to many species (Bell, 2012; Kim et al., 2017). The population $x_{3}$ initially only feeds upon $x_{1}$ as a herbivore, defined by $a_{31}<0$, and competes for this resource with $x_{2}$, defined by $a_{32}>0$. However, $x_{3}$ 's metabolism provides the potential for it to feed upon $x_{2}$ via $\rho a_{32}$, and to change its preference for feeding between $x_{1}(\rho=0)$ and $x_{2}(\rho=1)$. Consumption of $x_{2}$ by $x_{3}$ is defined by $a_{32}<0$ with $\rho \neq 0$. The establishment of a new trophic level via the emergence of carnivory in this model is indicated by $a_{32}$ changing sign from positive to negative when $\rho$ is non-zero. The dominance of carnivory as a feeding strategy for $x_{3}$ is indicated by $\rho \rightarrow 1$ when $a_{32}$ is negative. 
ways ouside the ecospace $E$, and are not of interest. The properties of the ecological equilibrium 330 points relevant to the analysis are:

- $C_{0}$ : all populations are extinct. This point should always be unstable in ecological models of interest. The condition for instability goes with the existence of an autotroph;

- $C_{1}$; the plant $x_{1}$ exists at its "carrying capacity" $0<x_{1}^{*}=r_{1} / a_{11}<1$, when the herbivore $x_{2}$ and the omnivore $x_{3}$ are extinct. This point should always be unstable as instability implies that a herbivore(s) can exist on the plant;

- $C_{4}$ : the plant $x_{1}$ and the herbivore $x_{2}$ coexist in a prey-predator relationship. This is ensured if $r_{2} / a_{21}<r_{1} / a_{11} . C_{4}$ must be unstable for the coexistence point $C_{7}$ to exist in ecological space;

- $C_{5}$ : the plant $x_{1}$ and the herbivore $x_{3}$ coexist. This is ensured if $r_{3} / a_{31}<r_{1} / a_{11}$. This point must be unstable for the coexistence point $C_{7}$ to exist in ecological space;

- $C_{7}$ : all three populations coexist.

The ecospace $E$ of the ecological sub-model (11) with the approximate locations of the equilibrium points and the boundary vector fields is shown in Figure 3

The equilibrium states of the evolutionary sub-model are:

$$
V_{0}=\left\{a_{32}^{*}>-a_{23}, \rho^{*}=0\right\} \quad \text { and } \quad V_{1}=\left\{a_{32}^{*}=-a_{23}, \rho^{*}=1\right\} .
$$

${ }_{345} V_{0}$ is a half-line and is sensitive to the evolutionary rate parameter $h_{\rho}^{2}$, as if $\rho \rightarrow 0$ "quickly enough" then $a_{32}^{*}$ could take any value between its initial value and $-a_{23}$.

\subsection{Equilibrium Stability}

The stabilities of the equilibrium points are determined by the eigenvalues of the Jacobian matrix (15), which is provided in Appendix B evaluated at each of the equilibrium points. This is shown in Appendix C. Note that the non-vanishing off-diagonal blocks of the Jacobian matrix show that the evolutionary processes affect the ecology and vice versa.

The equilibrium states of most interest in this model are the equilibrium half-line $\left\{C_{7}, V_{0}\right\}$ and the equilibrium point $\left\{C_{7}, V_{1}\right\}$. This latter point has complicated eigenvalues in the ecological space, but in general, $C_{7}$ exists in the ecospace and has eigenvalues with negative real parts if the boundary equilibrium points $C_{0}, C_{1}, C_{4}$, and $C_{5}$ are unstable. The parameter set the simulations start with has $C_{4}$ stable, so that $C_{7}$ is outside the ecospace, and if $x_{3}$ does not evolve it goes extinct. However, $x_{3}$ quite rapidly evolves, destabilising $C_{4}$ and bringing $C_{7}$ into the ecospace as an attracting point that determines the ultimate dynamical properties of the populations. The outcomes of the eco-evolution then are primarily determined by the eigenvalues in the evolution-

360 ary space. The eigenvalues of the coupled system (11) are in general analytically complicated and uninformative, but we can obtain explicit expressions for the evolutionary eigenvalues for the $\left\{C_{7}, V_{0}\right\}$ equilibrium half-line:

$$
\begin{aligned}
& \lambda_{4, C_{7}, V_{0}}=0, \\
& \lambda_{5, C_{7}, V_{0}}=h_{\rho}^{2}\left(\frac{1}{\beta_{\rho}}\right)\left(a_{31} x_{1}^{*}-a_{32}^{*} x_{2}^{*}\right) .
\end{aligned}
$$




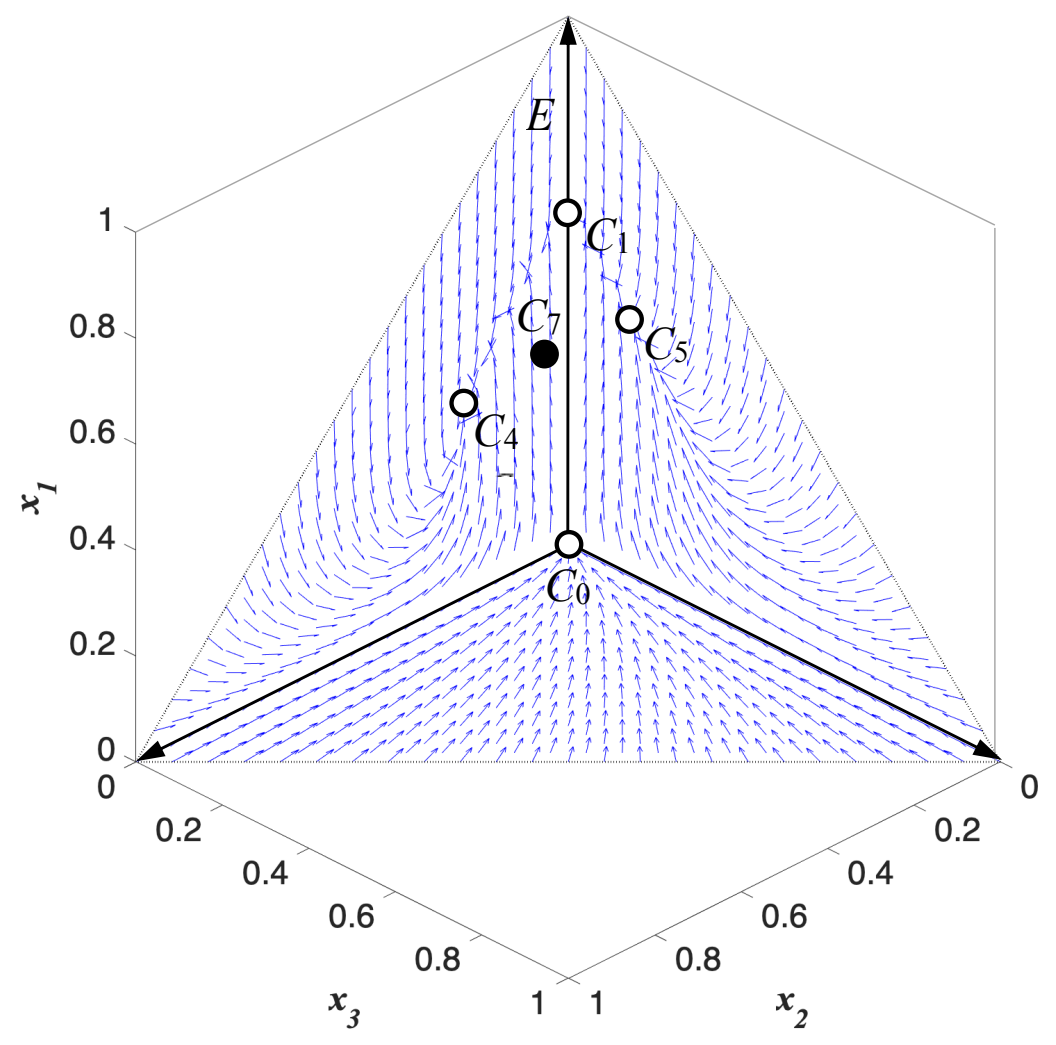

Figure 3: The ecospace for the ecological sub-model $(11)$. The unstable boundary equilibrium points $C_{0}, C_{1}, C_{4}$, and $C_{5}$, where one or more populations are zero, are shown as white dots, with the stable interior equilibrium point $C_{7}$, where all three populations coexist, shown as the black dot. Only solutions inside $E$, the corner of the $x_{1}, x_{2}, x_{3}$ cube where $x_{1}+x_{2}+x_{3} \leq 1$ are ecologically valid solutions to (11). The properties of (11) ensure that all solutions that begin in $E$ stay in $E$ for all time. Two-dimensional vector fields are shown on the boundary faces to give an indication of the vector field of $E$.

This half-line will have an attracting manifold when $a_{32}^{*}>a_{31} x_{1}^{*} / x_{2}^{*}$, which becomes a repelling manifold when $a_{32}^{*}<a_{31} x_{1}^{*} / x_{2}^{*}$. This change of stability suggests that the equilibrium point $\left\{C_{7}, V_{1}\right\}$, for which we are unable to obtain analytic expressions for the eigenvalues, may be stable and dominate the long-term dynamics of the system. Numerical simulations confirm that this is the case.

\section{Numerical Simulations}

Three cases are examined numerically to reveal the dynamics of the eco-evolutionary system. The cases of interest are:

- The case where the rate of adaptation of $\rho$ is much slower than the adaptation of $a_{32}$, say $h_{\rho}=0.10$, and $h_{\rho} / \beta_{\rho}=0.03$ versus $h_{a_{32}}^{2} / \beta_{a_{32}}=0.31$; 
- The case where the rate of adaptation of $\rho$ is approximately equal to the adaptation of $a_{32}$. Here, $h_{\rho}=0.15$, and $h_{\rho}^{2} / \beta_{\rho}=0.25$ is approximately equal to $h_{a_{32}}^{2} / \beta_{a_{32}}=0.31$;

- The case where the rate of adaptation of $\rho$ is much faster than the adaptation of $a_{32}$, say $h_{\rho}=0.20$, and $h_{\rho}^{2} / \beta_{\rho}=0.70$ versus $h_{a_{32}}^{2} / \beta_{a_{32}}=0.31$.

The model was integrated for 5,000 time units, which we will loosely refer to as "generations", from the initial conditions $\left\{x_{1}=0.25, x_{2}=0.25, x_{3}=0.25, a_{32}=0.10, \rho=0.25\right\}$. Note that the parameter set and initial conditions that we begin the simulations with will result in $x_{3}$ going extinct unless it modifies its initial feeding strategy. We also undertake a brief analysis of the sensitivity of the outcome to the relative rates of evolution. The parameters used to generate the Figures 4- 8 are provided in Table 1 in Appendix D Note that although the heritability $h^{2}$ of a trait may vary over time we fix these values during each simulation. However, we explore the influence of varying the heritability on the properties of the system.

\section{Results and Discussion}

The results of the first numerical simulation of equation (11) with the parameter set of Table 1. with $h_{\rho}=0.10$ (i.e. "slow" $\rho$ evolution) reveals in Figure 4 that $a_{32} \rightarrow-a_{23}$, so that $x_{3}$ develops the ability to consume $x_{2}$ very quickly. $x_{3}$ initially also improves its grazing on $x_{1}$ in a bid to stave off extinction, initially reducing $\rho$, but quickly abandons that strategy to become a carnivore, and maximises $\rho$. This change in feeding strategy is clearly evident in Figure 4 ff), where the dashed line shows that $x_{3}$ initially feeds exclusively on $x_{1}$, but then relatively slowly replaces its consumption of $x_{1}$ with predation on $x_{2}$, until by the end of the simulation it is almost exclusively a carnivore. The influence of the attracting manifold of the evolutionary equilibrium half-line $V_{0}$ is evident in the initial part of the simulation, before the system is eventually attracted to the evolutionary equilibrium point $V_{1}$. Note that the ecological equilibrium point $C_{7}$ appears stable throughout the latter part of the simulation.

The model simulation using an increased $\rho$ additive heritability, from $h_{\rho}=0.10$ to $h_{\rho}=$ 0.15 , (Figure 5) causes the evolution of $a_{32}$ to proceed more slowly, and counter-intuitively also slows down the transition of $x_{3}$ to carnivory. In this simulation, $x_{3}$ still quite rapidly increases its grazing on $x_{1}$, but remains a pure herbivore for much longer than previously, beginning to transition to carnivory at about generation 1,000, rather than 500 as in Figure 4. Although the onset of the transition to carnivory is delayed, once the transition initiates it proceeds more rapidly, with the final value of $\rho$ in the $h_{\rho}=0.15$ simulation exceeding the final value of the $h_{\rho}=0.10$ simulation. Again, the ecological equilibrium point $C_{7}$ appears stable throughout the simulation, while the evolutionary equilibrium half-line $V_{0}$ and point $V_{1}$ control the evolution of the system.

Further increasing the value of $\rho$ 's additive heritability, to $h_{\rho}=0.20$ (Figure 6), produces a similar slowing of the onset of the transition to carnivory, with the transition now not initiating during the 5,000 generations simulated. Further, the maximisation of $x_{3}$ 's functioning as a herbivore is also restricted, with $a_{32}$ stalling at approximately $a_{32}=-0.075$, well short of its potential minimum of $a_{32}=-a_{23}=-0.25$. In this case, the system trajectory has become trapped near the $V_{0}$ equilibrium half-line, and it is likely that $x_{3}$ will go extinct before it can transition to carnivory. Here, the influence of the attracting manifold of the evolutionary equilibrium half-line $V_{0}$ is evident in the initial part of the simulation, and appears to be stable, but as $a_{32}<0$ the eigenvalue (13) is positive so if $x_{3}$ managed to survive what appears will be a very long evolutionary bottle-neck, it could eventually transition to carnivory. However, the reduced feeding by 

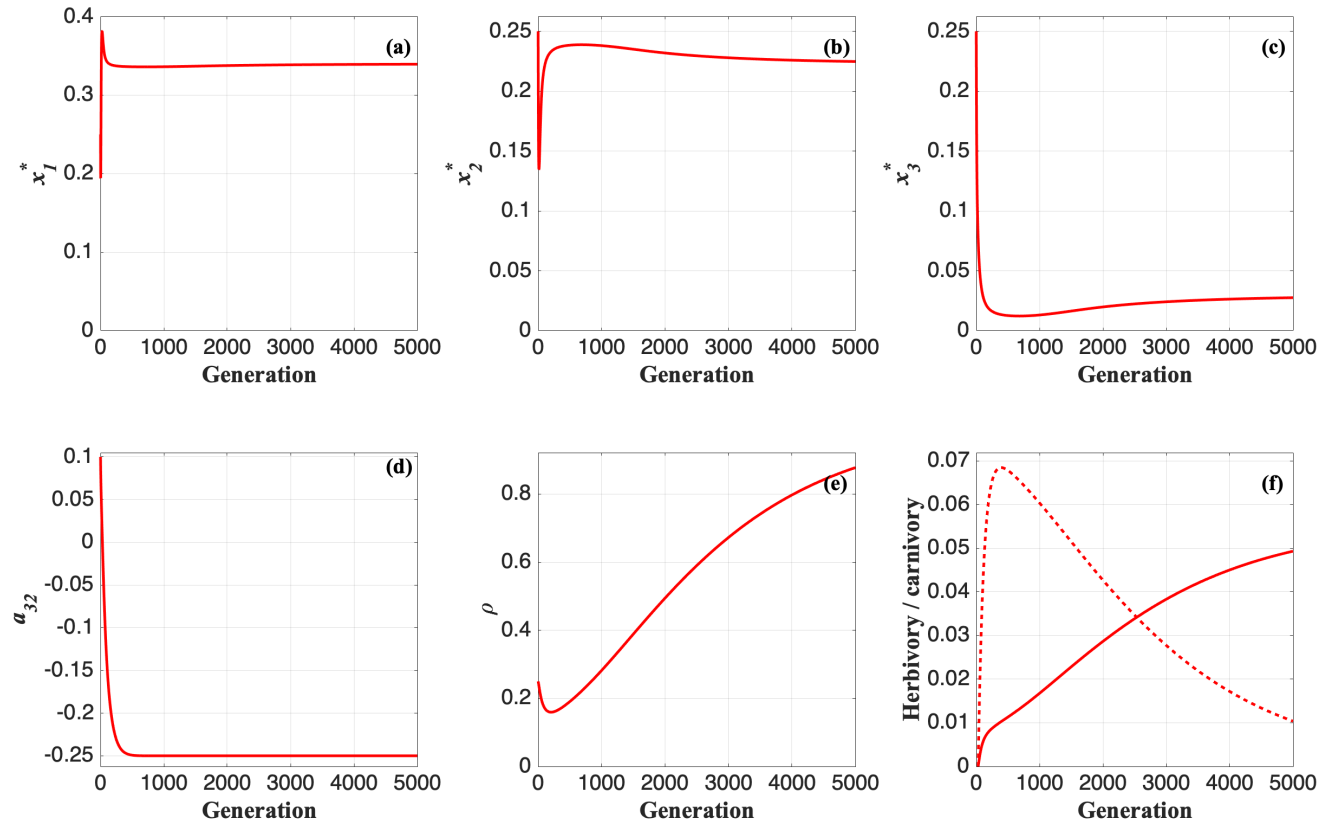

Figure 4: Simulation results for the populations $x_{1}(\mathrm{a}), x_{2}(\mathrm{~b})$, and $x_{3}(\mathrm{c})$, the values of the parameters $a_{32}(\mathrm{~d})$, and $\rho$ (e) from the model (11, and $x_{3}$ 's feeding (f) on $x_{1}$ (dashed line) and $x_{2}$ (solid line). Initial conditions are $\left\{x_{1}=0.2, x_{2}=\right.$ $\left.0.2, x_{3}=0.2, a_{32}=0.1, \rho=0.25\right\}$ with $h_{\rho}=0.10$. The initial response of $x_{3}$ to its parlous initial state (i.e. approximately the first 500 generations) is to improve its functioning as a herbivore, while rapidly developing its ability to function as a carnivore. It then adapts from herbivory to carnivory over generations $500-5,000$. This is the "slow" $\rho$ evolution case.

herbivory due to the truncated evolution of $a_{32}$ suggests that $x_{3}$ will go extinct, and the stable ecological equilibrium point $C_{7}$ will leave the ecospace via a transcritical bifurcation with $C_{4}$.

Figure 7 provides a view of the interplay between the mean value and the variance of the trait $\rho$. Panels (a) and (b) of Figure 7 show the evolution of the mean (a) and variance (b) of $\rho$ during the simulation. The initial variance reduces as $\rho$ approaches its lower bound, and evolution slows, however, once the unstable manifold of the evolutionary equilibrium half-line $V_{0}$ influences the dynamics, the variance increases as $\rho$ transitions to its upper bound. As it nears this, evolution again slows down. However, Figure 7 (a) and (b) clearly show that $\rho$ is still evolving at the ef the simulation. Panels (c) and (d) of Figure 7 show a similar scenario to panels (a) and (b), although in this case the evolution of $\rho$ is slowing and has nearly stopped by the end of the simulation.

Panels (e) and (f) of Figure 7 show a different evolutionary outcome to the others. In this case, $\rho$ has approached its lower bound very closely, and its variance has therefore approached zero,

430 slowing further evolution dramatically. This is a rather counter-intuitive result, as increasing the additive heritability of $\rho, h_{\rho}^{2}$ could reasonably be expected to speed up its transition to carnivory. 

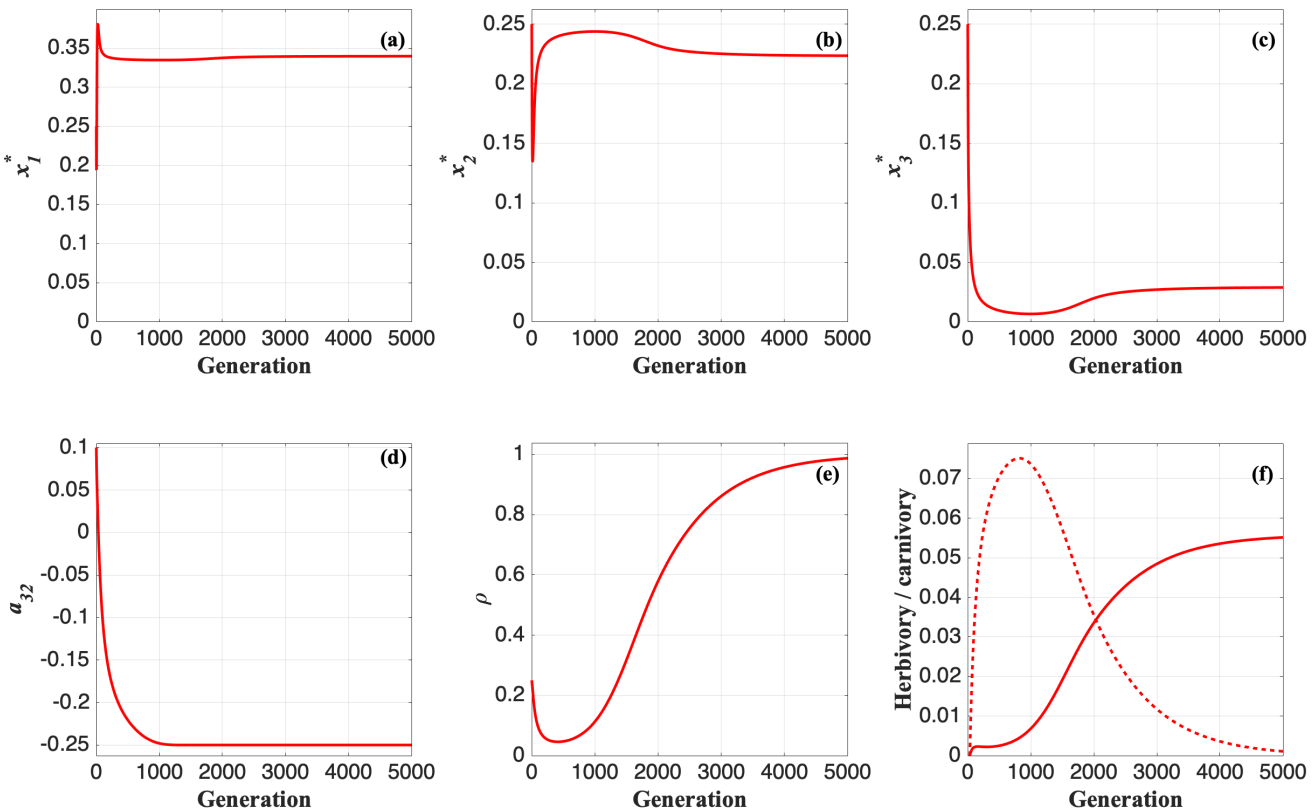

Figure 5: Simulation results for the populations $x_{1}$ (a), $x_{2}(\mathrm{~b})$, and $x_{3}$ (c), the values of the parameters $a_{32}$ (d), and $\rho$ (e) from the model (11, and $x_{3}$ 's feeding (f) on $x_{1}$ (dashed line) and $x_{2}$ (solid line). Initial conditions are $\left\{x_{1}=0.2, x_{2}=\right.$ $\left.0.2, x_{3}=0.2, a_{32}=0.1, \rho=0.25\right\}$ with $h_{\rho}=0.15$. The model was integrated for 5,000 time steps. The initial response of $x_{3}$ to its parlous initial state (i.e. approximately the first 1,000 generations) is to improve its functioning as a herbivore, while rapidly developing its ability to function as a carnivore. In this "intermediate" $\rho$ evolution case $x_{3}$ adapts from herbivory to carnivory over generations $1,000-5,000$ after recovering from near extinction.

As Figure 8 shows, this is not the case, and the onset of the transition is slowed by increasing the heritability.

The sensitivity of the population fitness to the average trait value $\frac{\partial f}{\partial \bar{z}}$ in the Lande (1976) equation determines the direction of evolution of each trait, but the factors determining the rates of evolution are less distinct. It is tempting to suggest that although the variance of the trait distribution, generically $\left(z_{U}-\bar{z}\right)\left(\bar{z}-z_{L}\right) / \beta\left(z_{U}-z_{L}\right)$, often is eventually the reason evolution stops, it is the additive heritability $h^{2}$ of the trait that essentially determines the rate of evolution of the trait. However, this is sometimes not as intuitive as it appears. The sensitivity of the rate of change of the feeding preference $\rho$ to its additive heritability $h_{\rho}^{2}$ is shown in Figure 8 This shows that increasing $h_{\rho}^{2}$ slows the rate of change of $\rho$, with $x_{3}$ functioning as a herbivore for longer, and constrains the evolution of the ability of $x_{3}$ to consume $x_{2}, a_{32}$ by reducing its negativity.

Finally we compare the rotation of the $f_{x_{3}}=0$ isocline in the simulations (Figure 9 ) with the behaviour predicted by our simple heuristic approach in $\$ 2$ in Figure 2 Initially, in panel (a), the coexistence equilibrium point $C_{7}$ is outside the ecospace, indicating that $x_{3}$ will not survive unless it evolves to bring $C_{7}$ into the ecospace $E$. After 500 generations (b) $x_{3}$ has developed the 

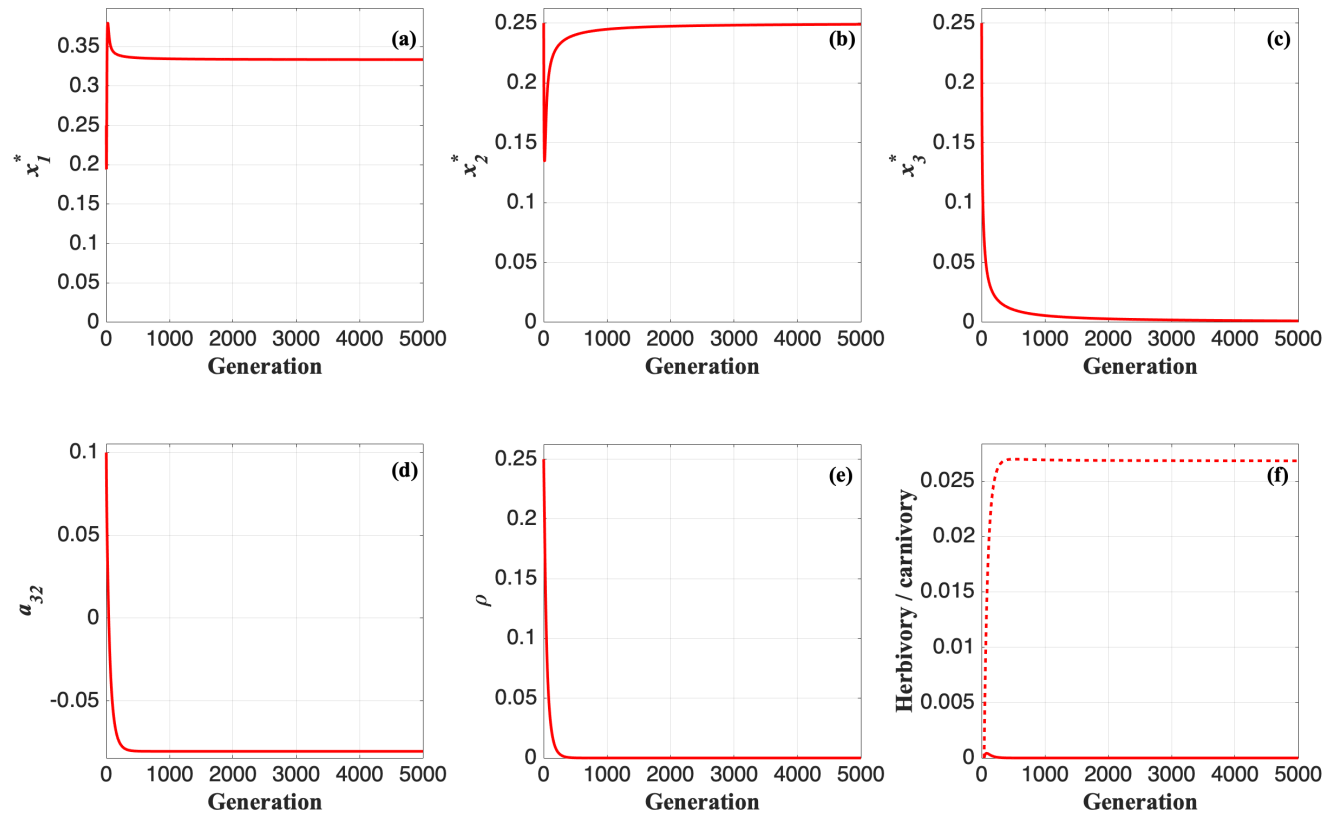

Figure 6: Simulation results for the populations $x_{1}$ (a), $x_{2}$ (b), and $x_{3}$ (c), the values of the parameters $a_{32}$ (d), and $\rho$ (e) from the model (11, and $x_{3}$ 's feeding (f) on $x_{1}$ (dashed line) and $x_{2}$ (solid line). Initial conditions are $\left\{x_{1}=0.2, x_{2}=\right.$ $\left.0.2, x_{3}=0.2, a_{32}=0.1, \rho=0.25\right\}$ with $h_{\rho}=0.20$. The model was integrated for 5,000 time steps. In this "fast $\rho$ evolution" case $x_{3}$ remains a herbivore and effectively goes extinct.

ability to predate on $x_{2}\left(a_{32} \approx-0.225\right)$, and its zero isocline has started to rotate anti-clockwise. However, it still feeds exclusively on $x_{1}(\rho \approx 0)$. This adaptation places $C_{7}$ on the boundary of $E$, a position that still results in the extinction of $x_{3}$.

After 1,000 generations (Figure $9 \mathrm{k}$ ), $x_{3}$ begins its transition to carnivory. $C_{7}$ has moved into the ecospace, and the $f_{x_{3}}=0$ isocline continues to rotate. After 5,000 generations, $x_{3}$ has effectively completed its transition to carnivory $(\rho \approx 1)$ and $C_{7}$ is well ensconced in the ecospace, ensuring $x_{3}$ 's survival. This completes the evolution $\rho$ and the anti-clockwise rotation of the $f_{x_{3}}=0$ ceases. The only option for $x_{3}$ to further improve its equilibrium population size is to slide its isocline further from its point of maximum resource, in this example $x_{2}=0.67$. This may be done by reducing its mortality rates $r_{3}$ and $a_{33}$, for which this example model does not include evolutionary equations.

\section{Conclusions}

The simulation of the evolution of a new trophic level, where a herbivore adopts carnivory instead, confirms the rubric arising from the consideration of the general properties of eco- 

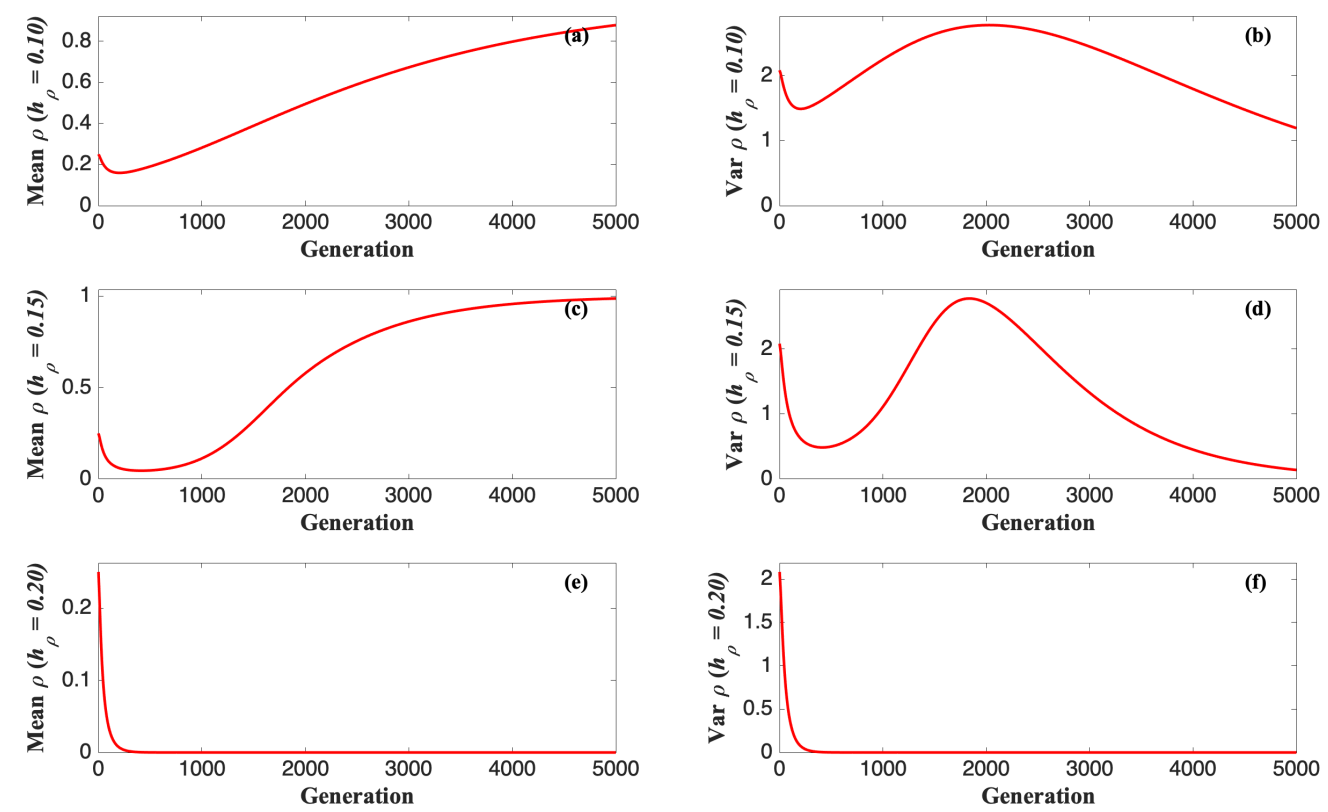

Figure 7: Means and variances of $\rho$ for the three simulations: (a) mean of $\rho$ for $h_{\rho}=0.10$; (b) variance of $\rho$ for $h_{\rho}=0.10$; (c) mean of $\rho$ for $h_{\rho}=0.15$; (d) variance of $\rho$ for $h_{\rho}=0.15$; (e) mean of $\rho$ for $h_{\rho}=0.20$; (f) variance of $\rho$ for $h_{\rho}=0.20$.

evolution in a resource-based framework. That is, in the presence of a dominant [or strong/stable/surviving ] competitor, it is better to learn to eat that competitor than to continue competing, losing, and possibly going extinct. A key factor in these results is the articulation of the ecosystem model in a consistent resource-based framework, and the requirement that our ecosystem models comply with fundamental ecological axioms. These insist that populations cannot be immortal, that is they cannot grow if they have no resources; and that populations must be viable, that is, they must be able to grow when maximum resource is available.

The transition from competition through omnivory to carnivory proceeds in this model in an orderly fashion, with no "exotic" behaviour. However, recent work by Gibert and Yeakel (2019) using a nonlinear omnivory model with carrying capacity revealed that complex dynamics may occur in the transition phase. They identified the potential for classic, inverted, and wasp-waisted distributions of biomass among trophic levels. The absence of complex dynamics in our simple heuristic model is most likely due to its linearity, as the Conservative Normal framework is able to preserve chaotic attractors observed in similar models that are constructed using carrying capacity (Cropp and Norbury, 2012). As most ecosystems function in the linear space of their nonlinear functional forms, exotic evolutionary transition dynamics may be relatively rare.

We can articulate some general rubrics from consideration of eco-evolutionary systems constructed in the Conservative Normal framework: 

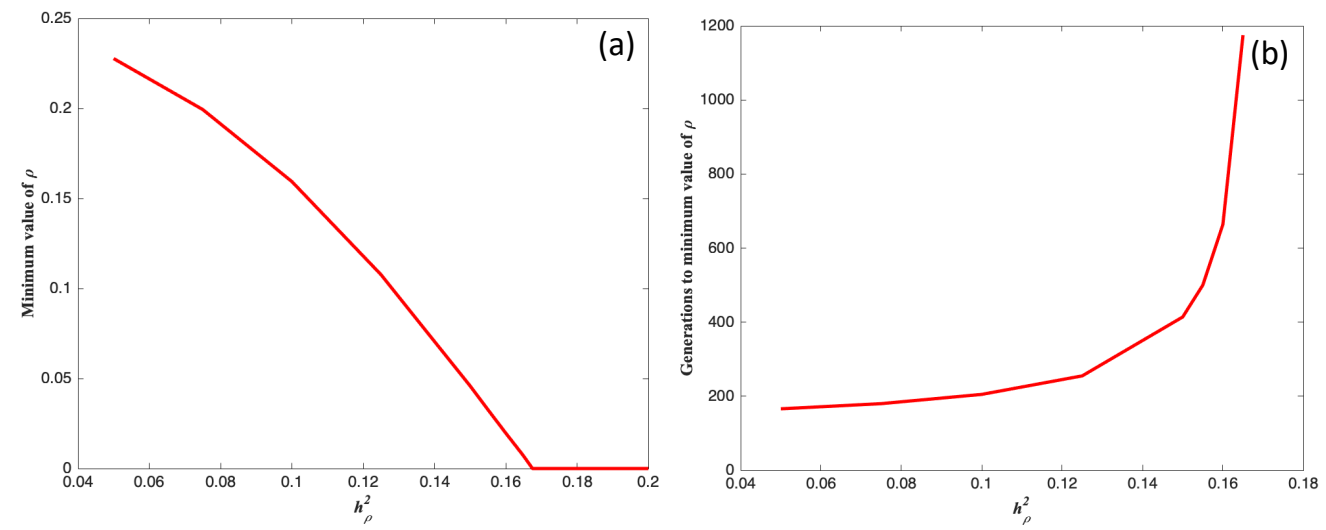

Figure 8: The effects of variations in the additive heritability of the feeding preference $h_{\rho}^{2}$ on the evolution of the ability of the omnivore $x_{3}$ to become a carnivore and feed upon $x_{2}$ are shown: (a) the effect of $h_{\rho}^{2}$ on the minimum value of $\rho$ (maximum level of herbivory) achieved before $x_{3}$ abandons herbivory to become a carnivore; and (b) the number of generations that $x_{3}$ optimises its function as a herbivore before abandoning herbivory to become a carnivore. Simulations as for Figures above.

- The bounds on the values that trait means can take, that are fundamental to the dynamics and equilibrium states of the eco-evolutionary systems, arise from fundamental ecological constraints, or axioms, imposed by the $\mathrm{CN}$ rules that ensure population viability, no immortality, and that fundamental principles such as conservation of mass of the key limiting resource cycling in the food web are respected;

- The $\mathrm{CN}$ eco-evolutionary equations using the life functions $f_{i}$ as a measure of fitness $\ln \bar{W}_{i}=f_{i}$ provide a simple rubric for the behaviour of populations in eco-evolutionary simulations - a population will seek to maximise the region of the ecospace in which it can grow (i.e. $f_{i}>0$ ). Put simply, every population seeks to move its zero growth isocline as far from its point(s) of maximum resource as possible;

- An implication of the above observation is that in any competition scenario, the maximum potential benefit to a competitor is obtained by evolving from competing for a resource to making its competitor a resource and predating upon it. This is a "safe" strategy for a population, as it may be achieved while maintaining a stable equilibrium at the same level as when it was a competitor, but which may be improved once it becomes a predator. This suggests that the development of new trophic levels may be a fundamental property of the eco-evolutionary models;

- Eco-evolutionary systems that include predation tend to have equilibrium half-lines in trait space in addition to, or instead of, equilibrium points. These half-lines mean that, although the ecological system appears to be in equilibrium, the trait means are evolving in a slow but significant manner that eventually may change the ecological state significantly. The outcomes of eco-evolutionary processes may then be sensitive to the rate parameters in the system, in particular the additive heritability parameter $h_{i}^{2}$; 

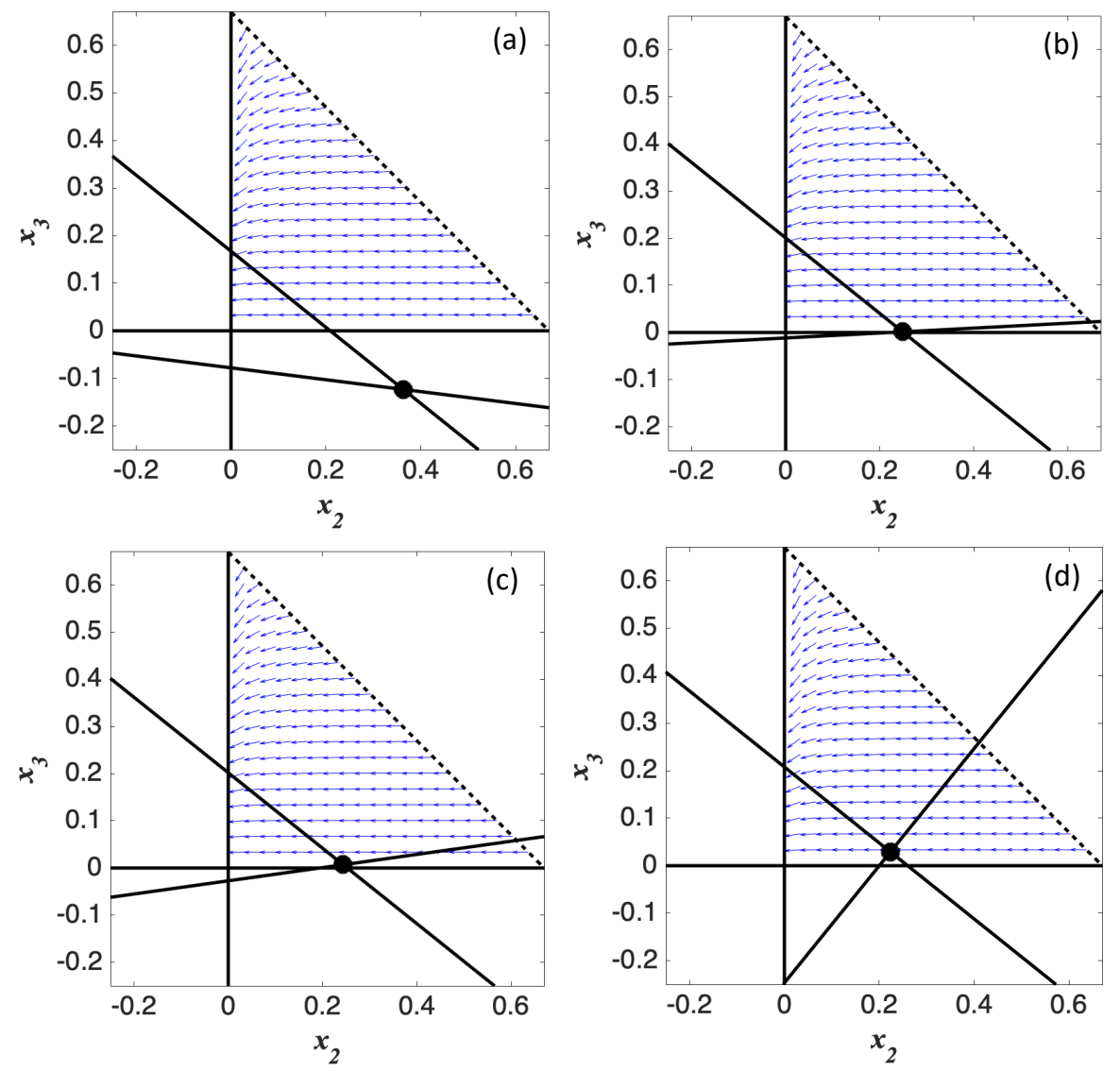

Figure 9: The rotation of the $f_{x_{3}}=0$ isocline in simulations with $h_{\rho}=0.15$ (Figure 5). Parts of all quadrants are shown, but only the region with the ecospace $E$ identified as the region with the blue arrows. These arrows show how the $x_{2}$ and $x_{3}$ populations' dynamics and equilibrium states change during the simulations. The resolution of the diagram does not allow the arrows that show the dynamics orbit $C_{7}$ in (c) and (d). The $f_{x_{2}}=0$ isocline does not move in these simulations. The black dot is the location of the coexistence equilibrium point $\left\{C_{7}, V_{1}\right\}$. As this is a two-dimensional slice through a three-dimensional vector field taken at $x_{1}=0.33, x_{2}$ and $x_{3}$ have maximum possible values of 0.67 . (a) The isoclines at the start of the simulation. Note $C_{7}$ is outside the ecospace, so without evolution $x_{3}$ will go extinct. (b) The isoclines after 500 generations. $x_{3}$ has evolved the ability to feed on $x_{2}\left(a_{32} \approx-0.225\right)$ but still feeds almost exclusively on $x_{1}$ $(\rho \approx 0)$. $x_{3}$ 's survival remains marginal. (c) After 1,000 generations $x_{3}$ begins its transition to carnivory. (d) After 5,000 generations, $x_{3}$ has almost completed its transition to carnivory $(\rho \approx 1)$, the $f_{x_{3}}=0$ isocline has rotated to an orientation typical of a predator, and $C_{7}$ is well within the ecospace ensuring $x_{3}$ 's survival.

- A consequence of the assumption of $\gamma$-distributed traits is that the variance of a trait in a population tends strongly to zero as the population approaches its evolutionary equilibrium for that trait. The implication of this property is that measurements of relative trait variances in real populations may provide indications of their distance from their evolutionary equilibrium, and hence provide indicators of the trait's additive heritability and/or the time the population has had to adapt the trait to its current environment. 
This work has assumed that the growth function $f_{x_{i}}$ is an appropriate measure of a population $x_{i}$ 's fitness, that naturally bounded traits are $\gamma$-distributed, and simulates the evolution of only the first and second moments of the trait distributions. Other bounded trait distributions might be assumed (Klauschies et al., 2018), other moments may be evolved (Coutinho et al., 2016), and other fitness functions could be used (Abrams et al., 1993, Fussmann et al., 2003; Cortez 2016, Pastor, 2017). Similarly, we have considered only the influence of the theoretical bounds - in practice, the theoretical bounds will be modified by physiological and environmental buffers that prevent the theoretical bounds from being reached. However, the possibility that a physiological bound lies outside a related system bound cannot be discounted. Notwithstanding these constraints, the heuristic considerations of $\$ 2$ suggest that the rubric arising from this analysis, that in the presence of a robust competitor it is better to adapt so as to eat the competitor, than it is to continue competing, is robust. In short, when faced with strong competition, "it is better to 520 eat than compete".

\section{Acknowledgements}

RC thanks the Mathematical Institute of the University of Oxford for financial support to facilitate this research, and thanks Lincoln College, Oxford for assistance with accommodation. The authors thank two anonymous referees and the editor for their suggestions for clarifications.

\section{Appendix A Ecological Equilibrium Points}

The equilibrium points of the ecological sub-model are:

$$
\begin{array}{cc}
C_{0}: & \left\{x_{1}^{*}=0, x_{2}^{*}=0, x_{3}^{*}=0\right\}, \\
C_{1}: & \left\{x_{1}^{*}=\frac{r_{1}}{a_{11}}, x_{2}^{*}=0, x_{3}^{*}=0\right\}, \\
C_{2}: & \left\{x_{1}^{*}=0, x_{2}^{*}=\frac{r_{2}}{a_{22}}, x_{3}^{*}=0\right\}, \\
C_{3}: & \left\{x_{1}^{*}=0, x_{2}^{*}=0, x_{3}^{*}=\frac{r_{3}}{a_{33}}\right\}, \\
C_{4}: & \left\{x_{1}^{*}=\frac{a_{22} r_{1}-a_{12} r_{2}}{a_{11} a_{22}-a_{12} a_{21}}, x_{2}^{*}=\frac{a_{11} r_{2}-a_{21} r_{1}}{a_{11} a_{22}-a_{12} a_{21}}, x_{3}^{*}=0\right\}, \\
C_{5}: & \left\{x_{1}^{*}=\frac{a_{33} r_{1}-a_{13} r_{3}}{a_{11} a_{33}-a_{13} a_{31}}, x_{2}^{*}=0, x_{3}^{*}=\frac{a_{11} r_{3}-a_{31} r_{1}}{a_{11} a_{33}-a_{13} a_{31}}\right\}, \\
C_{6}: & \left\{x_{1}^{*}=0, x_{2}^{*}=\frac{a_{33} r_{2}-a_{23} r_{3}}{a_{22} a_{33}-a_{23} a_{32}}, x_{3}^{*}=\frac{a_{22} r_{3}-a_{32} r_{2}}{a_{22} a_{33}-a_{23} a_{32}}\right\}, \\
C_{7}: & \left\{x_{1}^{*}=\frac{\mid A^{r} 1}{|A|}, x_{2}^{*}=\frac{\left|A_{2}\right|}{|A|}, x_{3}^{*}=\frac{\left|A^{r}\right|}{|A|}\right\},
\end{array}
$$

where $|A|$ denotes the determinant of the matrix of population interaction coefficients $a_{i j} \in A$ and $\left|A^{r_{i}}\right|$ denotes the determinant of the matrix of population interaction coefficients $A^{r_{i}}$ obtained by replacing column $i$ of the coefficient matrix $A$ with the column of environmental interaction

530 parameters $\tilde{r}=\left[r_{1}, r_{2}, r_{3}\right]^{\prime}$. Note that $C_{2}, C_{3}$ and $C_{6}$ are always outside the ecospace $E$ in this model and not of interest. This leaves the boundary equilibrium points $C_{0}, C_{1}, C_{4}$, and $C_{5}$, and the interior coexistence equilibrium point $C_{7}$ as the equilibrium points of interest in this model.

\section{Appendix B Jacobian Matrix}

The Jacobian matrix of the ecoevolutionary model $[11]$ is: 
$J=$

$\left[\begin{array}{ccccc}r_{1}-2 a_{11} x_{1}^{*} & -a_{12} x_{1}^{*} & -a_{13} x_{1}^{*} & 0 & 0 \\ -a_{12} x_{2}^{*}-a_{13} x_{3}^{*} & r_{2}-a_{21} x_{1}^{*} & -a_{23} x_{2}^{*} & 0 & 0 \\ -a_{21} x_{2}^{*} & -2 a_{22} x_{2}^{*}-a_{23} x_{3}^{*} & r_{3}-\left(1-\rho^{*}\right) a_{31} x_{1}^{*} & -\rho^{*} x_{2}^{*} x_{3}^{*} & a_{31} x_{2}^{*} x_{3}^{*} \\ -\left(1-\rho^{*}\right) a_{31} x_{3}^{*} & -\rho^{*} a_{32}^{*} x_{3}^{*} & -\rho^{*} a_{32}^{*} x_{2}^{*}-2 a_{33} x_{3}^{*} & -a_{32}^{*} x_{2}^{*} x_{3}^{*} \\ 0 & -h_{a_{32}}^{2}\left(\frac{a_{32}^{*}+a_{23}}{\beta_{a_{32}}}\right) \rho^{*} & 0 & -h_{a_{32}}^{2}\left(\frac{1}{\beta_{a_{3}}}\right) & -h_{a_{32}}^{2}\left(\frac{a_{32}^{*}+a_{23}}{\beta_{a_{32}}}\right) x_{2} \\ h_{\rho}^{2}\left(\frac{\left(1-\rho^{*}\right) \rho^{*}}{\beta_{\rho}}\right) a_{31} & -h_{\rho}^{2}\left(\frac{\left(1-\rho^{*}\right) \rho^{*}}{\beta_{\rho}}\right) a_{32}^{*} & 0 & -h_{\rho}^{2}\left(\frac{\left(1-\rho_{2}^{*}\right) \rho^{*}}{\beta_{\rho}}\right) x_{2}^{*} & h_{\rho}^{2}\left(\frac{1-2 \rho^{*}}{\beta_{\rho}}\right) \\ \left(a_{31} x_{1}^{*}-a_{32}^{*} x_{2}^{*}\right)\end{array}\right]$

${ }_{535}$ Appendix C Stability of Equilibria

The boundary equilibrium half-line $\left\{C_{0}, V_{0}\right\}$ and point $\left\{C_{0}, V_{1}\right\}$ have the eigenvalues:

$$
\begin{array}{ll}
\lambda_{1, C_{0}, V_{0}}=r_{1}>0, & \lambda_{1, C_{0}, V_{1}}=r_{1}>0, \\
\lambda_{2, C_{0}, V_{0}}=r_{2}<0, & \lambda_{2, C_{0}, V_{1}}=r_{2}<0, \\
\lambda_{3, C_{0}, V_{0}}=r_{3}<0, & \lambda_{3, C_{0}, V_{1}}=r_{3}<0, \\
\lambda_{4, C_{0}, V_{0}}=0, & \lambda_{4, C_{0}, V_{1}}=0, \\
\lambda_{5, C_{0}, V_{0}}=0, & \lambda_{5, C_{0}, V_{1}}=0,
\end{array}
$$

and are always unstable due to the presence of the autotroph $x_{1}$ which, as a viable population (we do not model unviable populations), always has $\lambda_{1, C_{0}}=r_{1}>0$.

The boundary equilibrium half-line $\left\{C_{1}, V_{0}\right\}$ and point $\left\{C_{1}, V_{1}\right\}$ have the eigenvalues:

$$
\begin{array}{ll}
\lambda_{1, C_{1}, V_{0}}=-r_{1}<0, & \lambda_{1, C_{1}, V_{1}}=-r_{1}<0, \\
\lambda_{2, C_{1}, V_{0}}=r_{2}-\frac{a_{21} r_{1}}{a_{11}}, & \lambda_{2, C_{1}, V_{1}}=r_{2}-\frac{a_{21} r_{1}}{a_{11}}, \\
\lambda_{3, C_{1}, V_{0}}=r_{3}-\frac{a_{31} r_{1}}{a_{11}}, & \lambda_{3, C_{1}, V_{1}}=r_{3}-\frac{a_{31} r_{1}}{a_{11}}, \\
\lambda_{4, C_{1}, V_{0}}=0, & \lambda_{4, C_{1}, V_{1}}=-h_{a_{32}}^{2}\left(\frac{1}{\beta_{a_{32}}}\right) x_{2}^{*} x_{3}^{*}<0, \\
\lambda_{5, C_{1}, V_{0}}=h_{\rho}^{2}\left(\frac{1}{\beta_{\rho}}\right) \frac{a_{31} r_{1}}{a_{11}}<0, & \lambda_{5, C_{1}, V_{1}}=-h_{\rho}^{2}\left(\frac{1}{\beta_{\rho}}\right) \frac{a_{31} r_{1}}{a_{11}}>0 .
\end{array}
$$

The boundary equilibrium point $\left\{C_{1}, V_{1}\right\}$ is unstable as $\lambda_{5, C_{1}, V_{1}}>0$ when $x_{3}$ is a herbivore, as then $a_{31}<0$. The stability of the equilibrium line $\left\{C_{1}, V_{0}\right\}$, will be determined by the signs of the $\lambda_{2, C_{1}, V_{0,1}}$ and $\lambda_{3, C_{1}, V_{0,1}}$ eigenvalues. As a general rubric, $\lambda_{2, C_{1}, V_{0,1}}$ is positive (and $C_{4}$ exists on the boundary of $E$ ) if the $f_{2}$ zero isocline intersects the $x_{1}$ axis between the origin and the $C_{1}$ point 
$r_{1} / a_{11}$. Similarly, $\lambda_{3, C_{1}, V_{0,1}}$ is positive (and $C_{5}$ exists on the boundary of $E$ ) if the $f_{3}$ zero isocline 545 intersects the $x_{1}$ axis between the origin and the $C_{1}$ point $r_{1} / a_{11}$.

The boundary equilibrium half-line $\left\{C_{4}, V_{0}\right\}$ has the eigenvalues:

$$
\begin{aligned}
2 \lambda_{1, C_{4}, V_{0}} & =-a_{11} x_{1}^{*}-a_{22} x_{2}^{*}+\sqrt{\left(a_{11} x_{1}^{*}+a_{22} x_{2}^{*}\right)^{2}-4 a\left(12 a_{21} x_{1}^{*} x_{2}^{*}\right.}, \\
2 \lambda_{2, C_{4}, V_{0}} & =-a_{11} x_{1}^{*}-a_{22} x_{2}^{*}-\sqrt{\left(a_{11} x_{1}^{*}+a_{22} x_{2}^{*}\right)^{2}-4 a\left(12 a_{21} x_{1}^{*} x_{2}^{*}\right.}, \\
\lambda_{3, C_{4}, V_{0}} & =r_{3}-a_{31} x_{1}^{*}-a_{32}^{*} x_{2}^{*}, \\
\lambda_{4, C_{4}, V_{0}} & =0 \\
\lambda_{5, C_{4}, V_{0}} & =h_{\rho}^{2}\left(\frac{1}{\beta_{\rho}}\right)\left(a_{31} x_{1}^{*}-a_{32}^{*} x_{2}^{*}\right) .
\end{aligned}
$$

For the parameter set used in the simulations (Table 1 , $\lambda_{3, C_{4}, V_{0}}>0$, so this line will always have an unstable manifold.

The boundary equilibrium point $\left\{C_{4}, V_{1}\right\}$ has the eigenvalues:

$$
\begin{aligned}
2 \lambda_{1, C_{4}, V_{1}} & =-a_{11} x_{1}^{*}-a_{33} x_{3}^{*}+\sqrt{\left(a_{11} x_{1}^{*}+a_{33} x_{3}^{*}\right)^{2}-4 a\left(13 a_{31} x_{1}^{*} x_{3}^{*}\right.}, \\
\lambda_{2, C_{4}, V_{1}} & =r_{2}-a_{21} x_{1}^{*}-a_{23}^{*} x_{3}^{*}, \\
2 \lambda_{3, C_{4}, V_{1}} & =-a_{11} x_{1}^{*}-a_{33} x_{3}^{*}-\sqrt{\left(a_{11} x_{1}^{*}+a_{33} x_{3}^{*}\right)^{2}-4 a\left(13 a_{31} x_{1}^{*} x_{3}^{*}\right.}, \\
\lambda_{4, C_{4}, V_{1}} & =0 \\
\lambda_{5, C_{4}, V_{1}} & =-h_{\rho}^{2}\left(\frac{1}{\beta_{\rho}}\right) a_{31} x_{1}^{*} .
\end{aligned}
$$

For the parameter set used in the simulations (Table 1 ) $\lambda_{2, C_{4}, V_{1}}>0$, so this point will always have an unstable manifold.

\section{Appendix D Parameters and Values}


Table 1: Parameter values used in equation (11) to draw the figures.

\begin{tabular}{lll}
\hline Parameter & Process & Value \\
\hline$r_{1}$ & Maximum $x_{1}$ autotroph growth rate & 1.00 \\
$r_{2}$ & Density-independent mortality rate of $x_{2}$ & -0.05 \\
$r_{3}$ & Density-independent mortality rate of $x_{3}$ & -0.05 \\
$a_{11}$ & Density-dependent mortality rate of $x_{1}$ & 1.50 \\
$a_{12}$ & Effect of $x_{2}$ predation upon $x_{1}$ & 2.00 \\
$a_{13}$ & Effect of $x_{3}$ predation upon $x_{1}$ & 1.50 \\
$a_{21}$ & Effect of $x_{1}$ food supply upon $x_{2}$ & -0.30 \\
$a_{22}$ & Density-dependent mortality rate of $x_{2}$ & 0.20 \\
$a_{23}$ & Effect of $x_{3}$ upon $x_{2}$ & 0.20 \\
$a_{31}$ & Effect of $x_{1}$ food supply upon $x_{3}$ & -0.15 \\
$a_{32}$ & Effect of $x_{2}$ upon $x_{3}$ & $-0.20 \rightarrow 0.10$ \\
$a_{33}$ & Density-dependent mortality rate of $x_{3}$ & 0.20 \\
$h_{32}$ & Realised additive heritability of $a_{32}$ & 0.25 \\
$h_{\rho}$ & Realised additive heritability of $\rho$ & 0.05 \\
$\beta_{a_{32}}$ & Shape parameter of the $a_{32} \gamma$-distribution & 0.20 \\
$\beta_{\rho}$ & Shape parameter of the $\rho \gamma$-distribution & 0.09 \\
$\rho$ & Feeding preference of $x_{3}$ & $0 \rightarrow 1$ \\
\hline
\end{tabular}

\section{References}

Abrams, P., 1999. The adaptive dynamics of consumer choice. Am Nat 153, 83-97.

555 Abrams, P., 2000. The evolution of predator-prey interactions: Theory and evidence. Annu. Rev. Ecol. Syst. 31, 79-105.

Abrams, P., Matsuda, H., 2004. Consequences of behavioral dynamics for the population dynamics of predator-prey systems with switching. Popul Ecol 46, 13-25.

Abrams, P., Matsuda, H., Harada, Y., 1993. Evolutionarily unstable fitness maxima and stable fitness minima of continuous traits. Evol Ecol 7, 465-487.

560 Bell, G., 2012. Experimental evolution of heterotrophy in a green alga. Evolution 67 (2), 468-476.

Birand, A., Barany, E., 2014. Evolutionary dynamics through multispecies competition. Theor. Ecol. 7, 367-379.

Bonhommeau, S., Dubroca, L., Le Pape, O., Barde, J., Kaplan, D., Chassot, E., Nieblas, A., 2013. Eating up the world's food web and the human trophic level. PNAS 110 (51), 20617-20620.

Chesson, P., 2000. Mechanisms of maintenance of species diversity. Annu. Rev. Ecol. Syst 31, 343-366.

565 Cortez, M., 2016. How the magnitude of prey genetic variation alters predator-prey eco-evolutionary dynamics. Am Nat $188,329-341$.

Cortez, M., Ellner, S., 2010. Understanding rapid evolution in predator-prey interactions using the theory of fast-slow dynamical systems. Am Nat 176 (5), E109-E127.

Cortez, M., Patel, S., 2017. The effects of predator-evolution and genetic variation on predator-prey population-level dynamics. B Math Biol 79, 1510-1538.

Coutinho, R., Klauschiies, T., Gaedke, U., 2016. Bimodal trait distributions with large variances question the reliability of trait-based aggregate models. Theor Ecol 9, 389-408.

Cropp, R., Norbury, J., 2012. Constructing ecologies. Journal of Theoretical Biology 294, 1-8.

Cropp, R., Norbury, J., 2015. Population interactions in ecology: A rule-based approach to modeling ecosystems in a mass-conserving framework. SIAM Rev 57 (3), 437-465.

Cropp, R., Norbury, J., 2019a. An eco-evolutionary system with naturally bounded traits. Theor Ecol, doi.org/10.1007/s12080-019-0407-6.

Cropp, R., Norbury, J., 2019b. The emergence of heterotrophy in an eco- evolutionary model: modelling trophic transitions in a resource-based framework with naturally-bounded trait distributions. Evol Ecol, doi.org/10.1007/s10682019-09981-4.

Cropp, R., Norbury, J., 2019c. Obligate mutualism in a resource-based framework. SIAM Review 61 (2), $596-622$. 
Edwards, K., 2019. Mixotrophy in nanoflagellates across environmental gradients in the ocean. PNAS 116 (13), 62116220.

Fisher, R., 1930. The genetical theory of natural selection. The Clarendon Press, Oxford.

585 Fussmann, G., Ellner, S., Jr., H. N., 2003. Evolution as a critical component of plankton dynamics. P R Soc London B Bio. 270, 1015-1022.

Gaedke, U., Klauschies, T., 2017. Analysing the shape of observed trait distributions enables a data-based moment closure of aggregate models. L\&O Methods 15, 979-994.

Gibert, J., Yeakel, J., 2019. Eco-evolutionary origins of diverse abundance, biomass, and trophic structures in food webs. Frontiers in Ecology and Evolution 7 (15), 1-11.

Hastings, H., Conrad, M., 1979. Length and evolutionary stability of food chains. Nature 282, 838-839.

Hawlena, D., Schmitz, O., 2010. Physiological stress as a fundamental mechanism linking predation to ecosystem functioning. American Naturalist 176, 537-556.

Kempthorne, O., 1957. An Introduction to Genetic Statistics. Iowa State Univ. Press, Ames, Iowa.

595 Kim, S., Cho, Y., Bhak, J., O’Brian, S., Yeo, J., 2017. Perspectives provided by leopard and other cat genomes: how diet determined the evolutionary history of carnivores, omnivores, and herbivores. BMB Reports 50 (1), 3-4.

Klauschies, T., Coutinho, R., Gaedke, U., 2018. A beta distribution-based moment closure enhances the reliability of trait-based aggregate models for natural populations and communities. Ecological Modelling 381, 46-77.

Kolmogorov, A., 1936. Sulla teoria di volterra della lotka per l'esisttenza. Giornale dell Instituto Italiano Degli Attuari 7, 74-80.

Kondoh, M., 2003. Foraging adaptation and the relationship between food-web complexity and stability. Science 299 , 1388-1391.

Kremer, C., Klausmeier, C., 2013. Coexistence in a variable environment: Eco-evolutionary perspectives. Journal of Theoretical Biology 339, 14-25.

605 Lande, R., 1976. Natural selection and random genetic drift in phenotype evolution. Evolution 30, 314-334.

Lande, R., 1979. Quantitative genetic-analysis of multivariate evolution, applied to brain-body size allometry. Evolution 33, 402-416.

Landi, P., Minoarivelo, H., Brannstrom, A., Hui, C., Dieckmann, U., 2018. Complexity and stability of ecological networks: a review of the theory. Population Ecology 60, 319-345.

610 Lindeman, R., 1942. The trophic-dynamic aspect of ecology. Ecology 23 (4), 399-417.

Loeuille, N., Loreau, M., 2005. Evolutionary emergence of size-structured food webs. PNAS 102 (16), 5761-5766.

May, R., 1972. Will a large complex system be stable? Nature 238, 413-414.

May, R., 1973. Stability and Complexity in Model Ecosystems. Princeton University Press, Princeton, New Jersey.

Merico, A., Brandt, G., Lan Smith, S., Oliver, M., 2014. Sustaining diversity in trait-based models of phytoplankton communities. Frontiers in Ecology and Evolution 2 (59), 1-8.

Merico, A., Bruggeman, B., Wirtz, K., 2009. A trait-based approach for downscaling complexity in plankton ecosystem models. Ecological Modelling 220, 3001-3010.

Nicholas, F., 1987. Veterinary Genetics. The Clarendon Press, Oxford.

Nuismer, S., 2017. Introduction to Coevolutionary Theory. W.H. Freeman, New York.

620 Odum, E., 1953. Fundamentals of Ecology, 1st Edition. W.B. Saunders Co, Philadelphia.

Osmond, M., Otto, S., Klausmeier, C., 2017. When predators help prey adapt and persist in a changing environment. Am Nat. 190, 83-98.

Pastor, J., 2017. Ecosystem ecology and evolutionary biology, a new frontier for experiments and models. Ecosystems $20,245-252$.

625 Post, D., 2002. The long and short of food-chain length. TRENDS in Ecology \& Evolution 17 (6), $269-277$.

Price, G., 1970. Selection and covariance. Nature 227, 520-521.

Price, G., 1972. Fisher's ‘fundamental theorem' made clear. Ann. Hum. Genet. 36, 129-140.

Romanuk, T., Binzer, A., Loeuille, N., Carscallen, W., Martinez, N., 2019. Simulated evolution assembles more realistic food webs with more functionally similar species than invasion. Scientific Reports 9 (18242).

630 Sahaney, S., Benton, M., 2008. Recovery from the most profound mass extinction of all time. Phil Trans Roy Soc B 275 , 759-765.

Svensson, E., Berger, D., 2019. The role of mutation bias in adaptive evolution. Trends in Ecology and Evolution 34 (5), $422-434$.

Terborgh, J., 2015. Toward a trophic theory of species diversity. PNAS 12 (37), 11415-11422.

Thompson, R., Brose, U., Dunne, J., Hall, R., Hladyz, S., Kitching, R., Martinez, N., Rantala, H., Romanuk, T., Stouffer, D., Tylianakis, J., 2012. Food webs: reconciling the structure and function of biodiversity. Trends in Ecology and Evolution 27 (12), 689-697.

Tilman, D., 1977. Resource competition between planktonic algae: an experimental and theoretical approach. Ecology $58,338-348$

640 Tilman, D., 1981. Tests of resource competition theory using four species of Lake Michigan algae. Ecology 62, 802-815. 
Vasseur, D., Amarasekare, P., Rudolf, V., Levine, J., 2011. Eco-evolutionary dynamics enable coexistence via neighbordependent selection. The American Naturalist 178 (5), E96-E109.

Vitousek, P., Matson, P., 2012. Nutrient cycling and biogeochemistry. In: Levin, S. (Ed.), The Princeton Guide to Ecology. Vol. 1. Princeton University Press, Princeton New Jersey, pp. 330-339.

645 Walsh, B., Lynch, M., 2018. Evolution and Selection of Quantitative Traits. Oxford University Press, Oxford. 